\title{
Successful Distance-based Training for Technologically Challenged Urban HIV Providers
}

\section{Downer G* \\ Howard University College of Medicine, USA}

*Corresponding author: Goulda Downer, Howard University College of Medicine, USA, 1840 7th Street NW, Second Floor, Washington DC 20001 Tel: 202-865-8146; Email: gdowner@howard.edu

\section{Mini Review}

Volume 2 Issue 2

Received Date: April 06, 2018

Published Date: May 04, 2018

DOI: $10.23880 /$ whsj- 16000113

\section{Abstract}

The Howard University College of Medicine implemented the AIDS Education and Training Center (AETC) Telehealth Training Center (HU-AETC TTC) was funded to use telehealth technologies to provide training and support to low volume HIV providers (i.e. those treating fewer than 10 patients who are HIV positive, per month). The program ran for three consecutive years and was carried out in Delaware, Maryland and Washington, DC. The major opportunities that were harnessed, i.e. lesson learned in regards to strengthening the Telehealth program is that needs assessment, not just knowledge of the subject matter but the infrastructure (time, equipment, support from management, staffing, ability to use technology, etc.) was key to the successful implementation and sustainability of a program such as this. Additionally, the success of our intervention was reinforced with our culturally competent, multi-factorial approach that addressed the individual and collective needs of the participants.

Keywords: HIV; Antiretroviral therapy; Epidemic; Effective

Abbreviations: AETC: AIDS Education and Training Center; PLWH: Persons Living with HIV; ART: Antiretroviral Therapy; HIVMA: HIV Medical Association's; AETC-NMC: AIDS Education and Training National Multicultural Center; NIAID: National Institute of Allergy and Infectious Disease.

Diagnosing persons living with HIV (PLWH), linking them to HIV primary care, and having patients achieve viral suppression are important public health steps toward ending the HIV epidemic. There is strong evidence that antiretroviral therapy (ART) [1] result in viral suppression, it prevents sexual HIV transmission and this underscores the importance of supporting effective interventions for linking to care; retaining in care, and adhere to antiretroviral therapy. Now, while there has been significant progress toward ending the HIV epidemic in the US, disparities still abound.

According to the HIV Medical Association's (HIVMA) [2] workforce and capacity survey clinics reported a difficulty in recruiting HIV care providers. This coupled with a shortage of well-trained HIV clinicians in the U.S. is attributed to several factors, including that HIV medicine 


\section{Women's Health Science Journal}

is not taught in many health professions academic or training programs because it is generally not required by school accrediting agencies and as a result young providers are choosing medical careers outside of HIV and primary health care. Also many first generation HIV clinicians are retiring from practice. This becomes problematic because the anticipated [3] HIV workforce shortage can result in more PLWH not in care and not successfully linked or retained thus increasing the risk of both HIV prevalence and incidence [2].

\section{Lack of Needs Assessment Data to Inform our Work}

Because agency needs dictated immediate program implementation and corresponding results, we were discouraged from distributing a needs assessment tool to assess infrastructure needs to support distancebased learning (webinar) [4] among participants. Based on the lack of this key information, we were unable to quickly identify the following and correct them during the first six months of the training program:

More than $61 \%$ clinicians reported that they did not have a dedicated computer in their offices in order to participate in the bi-weekly webinar sessions.

Most providers have a single generic email for their practice; thus the high likelihood of the receptionist reviewing email announcements and not forwarding them to the provider.

Ongoing technical assistance was needed since most providers were not technologically savvy and found registering online for the webinar sessions to be challenging. Instead, they opted to call in. This made it difficult for us to know who had fully participated in the training sessions and thus keep an accurate participant record log.

Among those clinics and private practitioners who were able to participate in the webinars, most had access to only one computer per facility with multiple team members gathering around one screen to receive trainings. This created difficulty from an evaluation standpoint since each provider is required to have an individual computer screen to interactively complete the pre/post questions. Additionally, if one participant registered with multiple participants viewing the webcast, this also decreased the likelihood of obtaining registration data for each provider.
Several providers who showed disinterest in the program shared that a lack of support from organizational directors as well as not having sufficient administrative support in their offices during the training times, were major barriers.

\section{Outcome}

Subsequently, we were able to conduct the needs assessment and obtain the requisite data thereby allowing for the development of a culturally appropriate infrastructure support system for participating clinicians. This client-focused system provided technical assistance on obtaining appropriate hardware, including computers for webinars participation. Technical assistance also included how to sign up for and then log on to live webinar trainings and also use the chat box during those training. Our efforts were aimed at encouraging participation by clinicians to increase their knowledge and skills about HIV prevention, management and treatment. Armed with this focused information, we developed and implemented a successful educational program using digital technology aimed at reducing HIV disparity in our region.

\section{Successful Program Output}

Increase in the numbers of providers trained was evidenced each year. Year 1: (75 persons trained); Year 2: (129 persons trained -reflecting a $172 \%$ increase); year 3 : 492 persons trained - reflecting $174381 \%$ increase over year 2 and $656 \%$ over year 1 ).

We were similarly successful in expanding the number. These health professionals work together with patients, family caregivers, and community service providers, etc. on shared goals within and across settings to achieve care that is safe, effective, patient-centered, timely, efficient, and equitable.

HU-CRTP participants consistently demonstrated increased knowledge throughout the funding cycle with on average 1 standard deviation of the mean.

Longitudinal impact studies: Baseline; 30; and 90 day follow-up surveys of all training participants to assess the effect of the training on the quality of the HIV services they provide; specifically whether they had applied the knowledge and skills gained in the training in their practice. The surveys were administered using Survey Monkey and the analysis indicates that $73 \%$ of trainees used training continued to use their practice more than 90 days after the training session. 


\section{Women's Health Science Journal}

Strategic business partnerships: Partnering with support from organizational directors/managers such as local health departments, particularly on individuals who had supervisory responsibility for providers, e.g., medical directors, clinic mangers, etc. facilitated provider recruitment. Additionally, archiving the trainings so that providers could take them when convenient was helpful in boosting recruitment and participation.

Development of a case study bank to accompany our trainings: Development of HU CRTP Case Study Bank, http://capitolregiontelehealth.org/case-studies/ was a key marketing tool. The bank which boasts a total of 48 case studies was used to support the seminars presented as part of the eRounds sections of our podcast series. Visitors to our site can access slides of each case study as well as full archived webinars in which they were presented along with a broader selection of On-The-Go Power Podcasts. Visitors to our site can also access additional Case Studies in Cultural Competency developed by the AIDS Education and Training - National Multicultural Center (AETC-NMC).

\section{Conclusion}

As in any period of innovation the rate of success depends more so on understanding the needs of the community to be served and not so much on the, "If you build it they will come" approach. The question is not so much whether digital learning is effective but whether the proposed program outcome is designed with need of the target population in mind. Furthermore, designing and implementing an effective digital learning program that is customized to the needs of the targeted population can yield positive lasting benefits.

\section{References}

1. National Institute of Allergy and Infectious Disease (NIAID). Preventing Sexual Transmission of HIV with Anti-HIV Drugs. In: Clinical Trials. gov. Bethesda (MD): National Library of Medicine (US).

2. NACO Annual Report Strategy to End AIDS in Fulton County.

3. The American Academy of HIV Medicine, HIV Specialist, Workforce Supply \& Demand.

4. A webinar is an educational, informative or instructional presentation that is made available online, usually as either video or audio with slides.

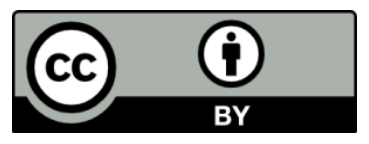

